\title{
Performance of Compressive Sensing Technique for Sparse Channel Estimation in Orthogonal Frequency Division Multiplexing Systems
}

\author{
P. Vimala ${ }^{1}$ and G.Yamuna ${ }^{2}$ \\ ${ }^{1}$ Assistant Professor, ${ }^{2}$ Professor, \\ Department of Electronics and Communication Engineering, \\ Faculty of Engineering and Technology, Annamalai University \\ Annamalai Nagar, Chidambaram, Tamil Nadu. \\ 1vimalakathirau@gmail.com \\ 2 yamuna.sky@gmail.com
}

\begin{abstract}
Orthogonal Frequency Division Multiplexing is a widely adopted multi carrier modulation in wireless communication systems due to its effective transmission and efficient bandwidth utilization ability. Wireless systems with coherent data detection require the estimation of channel at the receiver. Commonly employed pilot aided channel estimation probes the channel with known sequence called pilots and process the output to estimate the channel with linear reconsruction techniques like LS and MMSE. Wireless channels encountered in practice exhibits sparse structure that are having only a few dominant and many zeros coefficients. A recent development in Compressed Sensing (CS) has encouraged the extensive search on the application of sparse recovery algorithm to channel estimation. CS provides a constructive way to exploit the channel sparsity which reduces the number of pilots and hence increase spectral efficiency. Sparse channel estimation performed using sparse recovery algorithms provide better bit error rate compared with traditional LS and MMSE techniques. Further the quality of CS based sparse recovery algorithms depend on coherence of pilot structure therefore the pilot structure significantly affects the performance. To prove the efficacy of sparse recovery algorithm over pilot structure, performance of sparse recovery algorithm is evaluated for traditional combo and random pilot patterns generated. The random pilot with reduced mutual coherence has achieved better performance using sparse recovery algorithm.
\end{abstract}

Keywords - Compressed sensing, Coherence of Pilot Pattern, Orthogonal Frequency Division Multiplexing, Sparse Channel Estimation

\section{INTRODUCTION}

Orthogonal Frequency Division Multiplexing (OFDM) has been widely adopted due to its high data rate, efficient spectral utilization and ability to cope with multipath fading channel. Coherent reception in digital wireless systems, need to obtain channel impulse response accurately at the receiver [1]. Estimation of channel is a challenging problem in wireless communication systems. The two methods commonly employed to estimate the multipath channel at the receiver are pilot aided and blind. The pilot aided method estimates the channel by inserting known sequence called pilots into some prefixed positions of subcarriers. The corresponding channel outputs are processed to estimate the channel parameters at pilot subcarrier locations and employ interpolation techniques to estimate the channel at data subcarriers. Traditional pilot aided methods, often comprising of linear Least Squares (LS) and Minimum Mean-Square channel estimators (MMSE). It makes lower spectral efficiency and power utilization with buy back of quick response to the channel variation [2]. On the other hand, the blind method is based on the statistics of channels and requires a large number of received OFDM symbols to extract statistical properties. Furthermore their performance is worse than the pilot aided method. Therefore pilot aided channel estimation is widely employed in modern wireless communication systems. Depending upon the arrangement of pilots among the subcarriers, the popular pilot structures adopted in pilot aided channel estimation are block, combo and lattice type and among the pilot structures combo type yields better results by traditional channel estimation methods [3]-[4].

The new standards such as 3GPP Long Term Evolution (LTE) has been developed for higher bandwidth demands in mobile communication systems. These systems require comparatively large bandwidths, which make it possible to resolve the individual propagation paths from transmitters to receivers. This result in a channel impulse response with only few dominant channel coefficients and remaining coefficients are zero or approximately zero that is termed as sparse channel. Ttraditional pilot aided channel estimation methods rely on linear reconstruction methods and that are incapable to exploit the inherent sparsity of channels. The key idea of 
Compressive Sensing (CS) exploits the sparsity of the channels which improves the spectral and energy efficiency effectively by reducing the required number of pilots [5]. Advances in the field of CS have gained a much interest in signal processing and can be applied to sparse channel estimation. We conduct a comparative performance analysis for sparse channel estimation using traditional methods and CS reconstruction methods in terms of bit error rate using combo pilot structure. It is also analyzed that the efficiency of CS methods are based on pilot structure.

The remainder of the paper is organized as follows. Section 2 provides a description about the compressed sensing techniques. The section 3 provides about the OFDM system, frequency selective channel model and system model for spare channel estimation. The section 4 describes conventional and CS based channel estimation methods. The Section 5 presents the simulation of channel estimation and section 6 describes conclusion of the work.

\section{COMPRESSED SENSING THEORY}

The theory of CS extends the concept of traditional Nyquist sampling applicable only to band limited signals to broader class of signals. CS theory states that certain signals can be recovered from far fewer samples and measurements than traditional methods [6]. CS relies on two principles: sparsity and sufficient condition for recovery from sparse signals.

The measurement model of unknown signal vector $x \in R^{n}$ using matrix representation is given by

$$
y=A x
$$

where $y$ is measurement vector and $A$ is an $m \times n$ sensing matrix. Using the traditional linear measurement method, one needs $m$ measurements such that $m \geq n$. Theory of CS makes use of the fact that many natural signals have few non zeros entries or compressible to few non zeros and are referred as sparse. The sparsity expresses a key idea that the information rate of a continuous time signal may be much smaller than its suggested bandwidth. The sparse recovery from CS suggests that successful recovery of $x$ is possible even when $m$ is much smaller than $n$ if $x$ has few nonzero entries.

A sufficient condition guaranteeing the perfect recovery of the sparse signal $x$ from the model represented in equation (1) is expressed in terms of Restricted Isometry Property (RIP). A sensing matrix $A$ satisfies the RIP of order $\mathrm{K}$ if there exists a constant $\delta$ such that

$$
(1-\delta)\|x\|_{2}^{2} \leq\|A x\|_{2}^{2} \leq(1+\delta)\|x\|_{2}^{2}
$$

for any $K$ sparse vector $\left(\|x\|_{0} \leq K\right)$. The minimum of all constants $\delta$ satisfying the above condition is called isometry constant $\delta_{\mathrm{K}}$. But verifying a sensing matrix $A$ for RIP involves combinatorial computation complexity, other than RIP the widely used condition is mutual incoherence [7]. However $A$ satisfies the RIP incurs tremendous computational complexity because it does not have proper linear program. So an alternative to RIP and simple to calculate the condition for $A$ is the mutual coherence. The mutual coherence of a matrix $A$ is the largest absolute inner product between any two columns $a_{i}, a_{j}$ of $A$. It can be written as

$$
\mu(A)=\max _{1 \leq i<j \leq L}\left|\left\langle a_{i}, a_{j}\right\rangle\right|
$$

and a smaller value of $\mu(A)$, will lead to a more accurate recovery of $x$. Under above conditions of the matrix $A$, CS indicates that as long as the unknown signal $x$ is reasonably sparse, it is possible to recover $x$ by its reconstruction algorithms.

\section{III.SYSTEM AND CHANNEL MODEL}

OFDM is a multicarrier modulation scheme that allows large number of closely spaced orthogonal sub carrier signals to carry data on parallel data streams. Each sub carrier is modulated with conventional modulation scheme such as QAM or PSK at low symbol rate, maintaining total data rate similar to conventional single carrier modulation schemes in the same band width. The modulated data are inserted with pilots and converted into $N$ parallel data streams in which each sub-carrier consists of either data or pilot symbol. After implementing Inverse Fast Fourier Transform (IFFT), the $i^{\text {th }}$ discrete time domain OFDM symbol can be expressed as

$$
x_{i}(n)=\sum_{k=0}^{N-1} X_{i}[k] e^{\left(\frac{j 2 \pi k n}{N}\right)} \text { for } n=0,1,2, \ldots, N-1
$$

where $k$ represents the sub-carrier index. After adding cyclic prefix, the symbol $x_{t}$ is sent to a frequency selective multi-path fading channel and the received symbol in discrete domain can be represented as

$$
y_{t}(n)=x_{t}(n)^{*} h(n)+w(n)
$$


where $w(n)$ is the Additive White Gaussian Noise (AWGN) in time domain and $h(n)$ is the channel impulse response. At the receiver, after removing the cyclic prefix, the received symbols are demultiplexed using a FFT block by

$$
Y[k]=\frac{1}{N} \sum_{n=0}^{N-1} y(n) e^{\left(\frac{-j 2 \pi k n}{N}\right)}
$$

Then parallel data forms are converted into serial and demodulated. The channel is modeled as frequency selective and can be expressed as

$$
h(n)=\sum_{i=1}^{L} \alpha_{i} \delta\left(\left(n-\tau_{i}\right) T_{s}\right)
$$

where $\delta($.$) is a dirac delta function, L$ resolvable paths and each path has complex path gain $\alpha_{i}$ and delay spread $\tau_{i}$ with $T_{s}$ sampling interval. The ever increasing demands of data rate, leads to decrease the sampling interval $T_{s}$ compared to maximum delay spread of the channel which results in a channel with few dominant coefficients. The channel coefficient vector $h$ has only $K$ dominant coefficient, $h$ is $K$ sparse vector. CS techniques exploit the sparsity of such wireless channels [8]-[9].

Consider a pilot aided $N$ subcarriers OFDM system for sparse channel estimation. $N_{p}$ subcarriers used for pilot transmission and $N_{d}=N-N_{p}$ subcarriers used for data transmission among $N$ subcarriers. The pilot positions in subcarriers are represented as $\left[p_{1}, p_{2}, \ldots ., p_{N p}\right]$ where $1 \leq p_{1}<p_{2}<\ldots \ldots<p_{N p} \leq N$. According to CS, the estimation of sparse channel at the receiver can be modelled as [10]

$$
y=A . h+w
$$

where, $h=[h(1), h(2), \ldots, h(L)]^{\mathrm{T}}$ is channel impulse response with length $L, w=\left[w(1), w(2), \ldots, w\left(N_{p}\right)\right]^{\mathrm{T}}$ is an additive white Gaussian noise, $y=\left[y(1), y(2), \ldots, y\left(N_{p}\right)\right]^{\mathrm{T}}$ is a received pilot symbols among $N_{p}$ subcarriers of OFDM system. Sensing matrix $A=X . F_{N p \times L}$ where $X=\operatorname{diag}\left\{x\left(p_{1}\right), x\left(p_{2}\right), \ldots, x\left(p_{N_{p}}\right)\right\} \quad$ is diagonal matrix of transmit pilot symbols and $F_{N p \times L}$ is a discrete Fourier sub-matrix constructed by selecting $N_{p}$ row denoted by pilot location and $L$ columns of full discrete Fourier matrix. There is feasible solution from the theory of CS, if channel vector $h$ has only $K$ dominant coefficients such as $K<<L$ and rest of the coefficients are zero. For this case, the pilots are kept less than the channel coefficients $(N p<L)$ which significantly reduces pilots and improves the spectral efficiency. According to (3), if sensing matrix $A$ holds minimum coherence then, CS reconstruction algorithms yield efficient reconstruction therefore; pilot structure plays a major role in channel estimation using CS algorithms.

\section{IV.CHANNEL ESTIMATION TECHNIQUES}

The transmitted OFDM symbols over wireless communication channel are usually distorted by the channel characteristics. To compensate the channel effects at the receiver, the coherent detectors require knowledge of channel impulse response. The model represented in (8) is linear if $N_{p}>L$. The Least-Square (LS) and Minimum-Mean-Square-Error (MMSE) are popularly used traditional linear techniques for pilot-aided channel estimation in the OFDM systems at the receiver. LS is a low complexity channel estimation method however, without using any knowledge of the statistics of the channels. LS channel estimation can be represented as

$$
\hat{H}_{L S}=\left(X^{H} X\right)^{-1} X^{H} Y=X^{-1} Y
$$

Every component of the LS channel estimate at each subcarrier locations $k=0,1,2, . ., N-1$ can be written

$$
\hat{H}_{L S}[k]=\frac{Y[k]}{X[k]}
$$

Even though LS channel estimate suffers from a high MSE, it is widely used for channel estimation due to its simplicity [11]. To improve the LS channel estimation is updated using the weight matrix $W$ defined as

$$
\hat{H}_{M M S E}=W X^{-1} Y=W \widetilde{H}
$$

where $W=R_{H \widetilde{H}} R_{\widetilde{H} \widetilde{H}}^{-1}, R_{\widetilde{H} \widetilde{H}}$ is the auto correlation matrix of $\widetilde{H}$ and $R_{H \widetilde{H}}$ is the cross correlation between original channel vector and temporary channel estimate vector in frequency domain.

These traditional pilot aided channel estimation relies on combo type pilot arrangement since it is sensitive to frequency selectivity when comparing to the other arrangements. But these traditional techniques are more or less relying on linear reconstruction strategies and because of their dependence on linear procedure; the number of pilots used must be high. Regardless of choice of number of pilots, the reconstruction error is also more if the 
channel is sparse. The recently emerged CS exploits the inherent sparsity of the wireless fading channel and it depends on non linear reconstruction procedure. CS based methods have two main advantages of reducing pilot overhead and decreasing mean square error. If the knowledge of locations of nonzero taps is known to the structural LS estimator then it gives best estimate. But the receiver has no information about the nonzero coefficients location of $h$ therefore; structural LS estimator cannot be realized and CS based approach leads to optimal results. In pilot aided channel estimation, the pilot subcarriers occupy a fraction of spectrum but they do not carry any information and by reducing the number of pilot subcarriers, the spectrum efficiency can be increased. It is possible to estimate the channel coefficients with fewer numbers of pilots using CS based methods by considering the sparsity of channel.

The two main categories of CS based channel estimation methods are $l_{1}$ minimization and iterative greedy algorithms. The first method is to minimize the $l_{1}$ norm which is based on linear programming techniques. $l_{1}$ minimization provides accurate method for sparse signal recovery if sensing matrix $A$ satisfies RIP. But the computational complexity of $l_{l}$ minimization is highly impractical. It leads to need of faster recovery algorithm that works in linear time. Recently proposed several low complexity iterative greedy algorithms rely on iterative approximation of the signals either by identifying the support of the signal iteratively until a convergence criterion is met or by finding an improved estimate of the sparse signal in each of the iteration that attempts to account for the mismatch to the measured data. Some greedy methods actually have the performance guarantees that are equal to those obtained by convex optimization approaches. Two of the simplest and oldest greedy approaches are Orthogonal Matching Pursuit (OMP) and Iterative Thresholding. OMP is reliable for reconstructing both sparse and near sparse signals [12]. OMP iteratively detect and estimate the location and value of the channel from the measurement $y$ correctly with high probability. Iterative method is usually faster than $l_{1}$ minimization techniques in the orders of magnitude while they may fall short of performance. Iterative Thresholding make correct measurements by soft or hard thersholding from the noisy measurements of given sparse signal. The number of iterations and problem set up at hand defines the thresholding function. Sparse channel estimation in OFDM system is simulated using the iterative greedy OMP.

Simplicity and competitive reconstruction performance is the significance of OMP algorithm. The simplest guarantee for OMP states that for exactly $K$-sparse $h$ will be recovered exactly in $K$ iterations [13]-[14] if matrix $A$ satisfying either the RIP or coherence. The basic idea of OMP algorithm to recover the sparse signal is to select a column from $A$ at each iteration until it reach the sparse degree $K$. OMP starts by selecting a column of $A$ that is most correlated with the measurement $y$. This step of the algorithm is repeated by correlating the columns with the signal residual that is obtained by subtracting the contribution of a partial estimate of the signal from the original measurement vector. Algorithms contained in this category include Compressive Sampling Matching Pursuit (CoSaMP), Regularized Orthogonal Matching pursuit (ROMP) and Stagewise Orthogonal Matching pursuit (StOMP). Among all these algorithm OMP is reliable for reconstructing both sparse and near sparse signals.

\section{Simulation Results}

Pilot aided OFDM system is constructed using MATLAB version 7.12 tool with parameters furnished in Table I. Typical sparse channel with 5 dominant coefficients over maximum of 50 coefficients is given in Fig. 1.

TABLE I. System Parameters

\begin{tabular}{|l|c|}
\hline \multicolumn{1}{|c|}{ Parameters } & Value \\
\hline Number of Total Subcarriers & 256 \\
\hline Number of Pilot Subcarriers & 32 and 16 \\
\hline Number of Cyclic Prefix & 64 \\
\hline $\begin{array}{l}\text { Maximum Number of Channel } \\
\text { Coefficients }\end{array}$ & 50 \\
\hline $\begin{array}{l}\text { Number of Nonzero Channel } \\
\text { Coefficients }\end{array}$ & 5 \\
\hline Modulation & QPSK \\
\hline
\end{tabular}




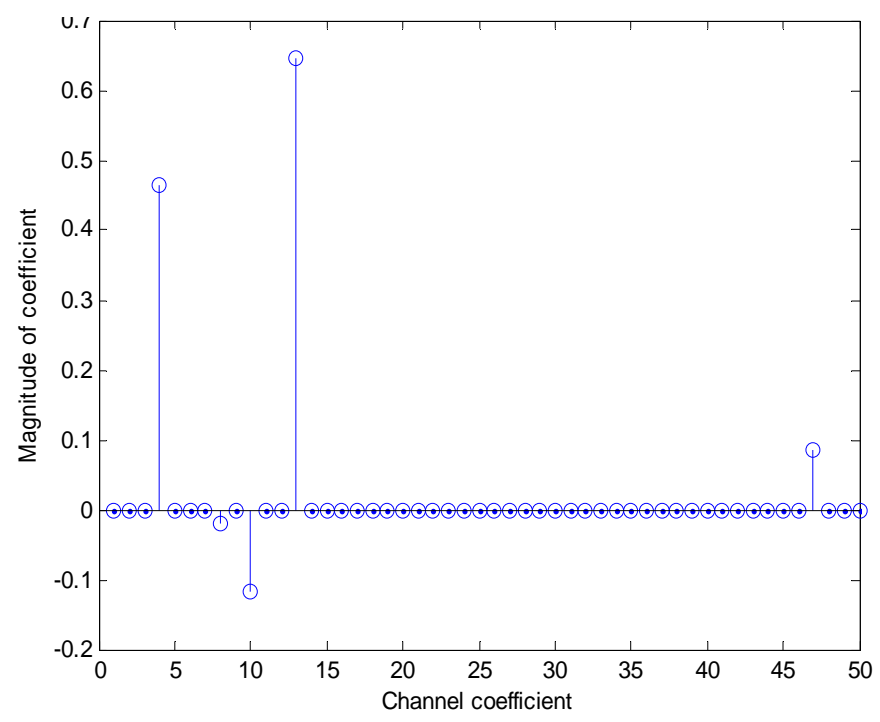

Fig. 1. Typical Sparse Channel

Sparse channel estimation is performed with traditional LS, MMSE and CS based OMP algorithm using conventionally best combo type pilots. Bit error rate performances of channel estimation are evaluated for pilot of length 16 and 32 for QPSK modulation scheme and are presented in Fig. 2 and 3. OMP algorithm achieves better performance when compared with conventional LS and MMSE algorithms, but bit error is only on the order of $10^{-0.08}$ and $10^{-0.9}$ in both the plots since the coherence of combo pilots is high. The performances of CS based methods are based on mutual coherence of the pilot structure therefore a random pilot structures are generated and the coherence of conventional combo pilot structure compared with random pilot structures and are presented in Fig. 4 as bar graph. The Fig. 5 shows the performance comparison for channel estimation with combo and random pilot structure of length 16 interms of bit error rate. The random pilot structure has achieved minimum bit error rate ranging about $10^{-3}$ for signal to noise ratio of $30 \mathrm{~dB}$ when compared with conventional combo pilot because of reduced coherence. CS algorithms provide better reconstruction performance for sparse channel if mutual coherence of the pilot structure is minimum.

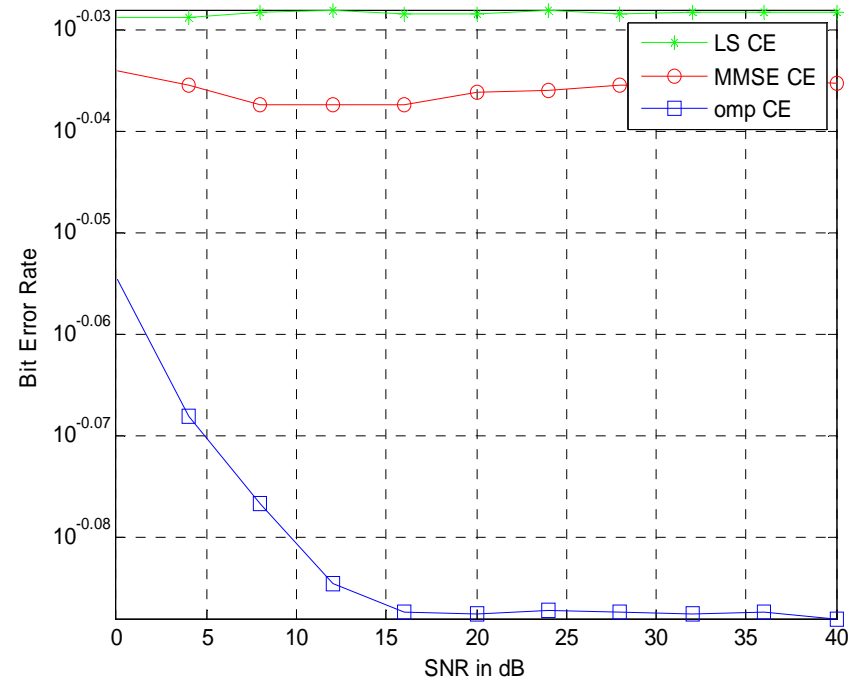

Fig. 2. Bit Error Rate Vs Signal to Noise Ratio for LS, MMSE and OMP Channel Estimations with 16 Pilots 


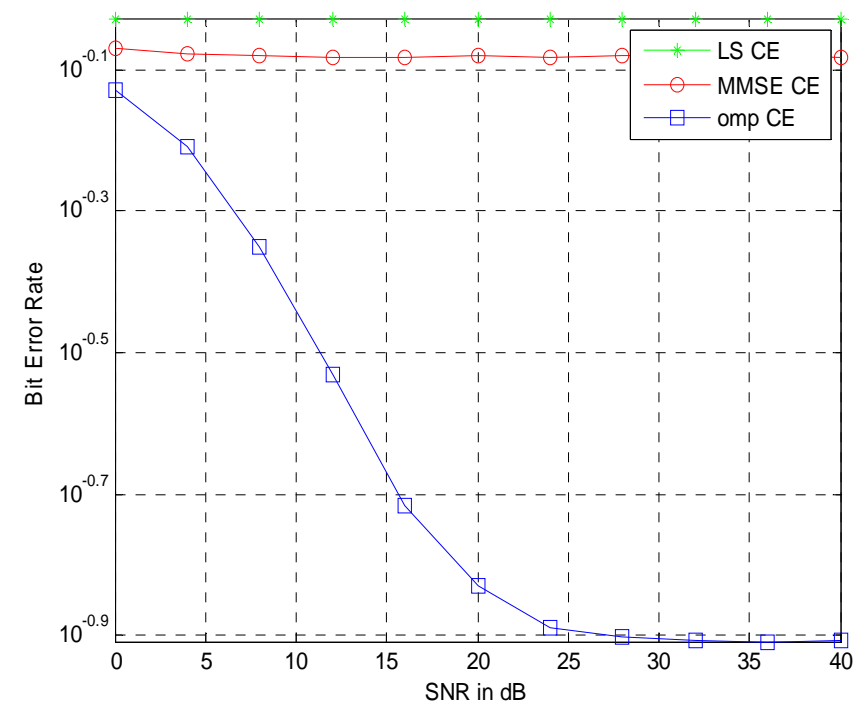

Fig. 3. Bit Error Rate Vs Signal to Noise Ratio for LS, MMSE and OMP Channel Estimations with 32 Pilots

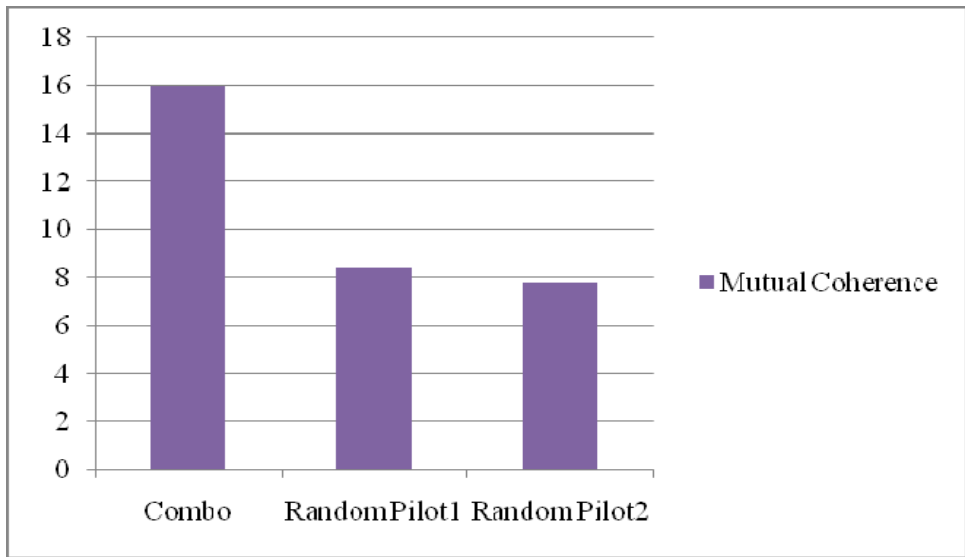

Fig. 4. Mutual Coherence Value for Combo and Random Pilot Patters

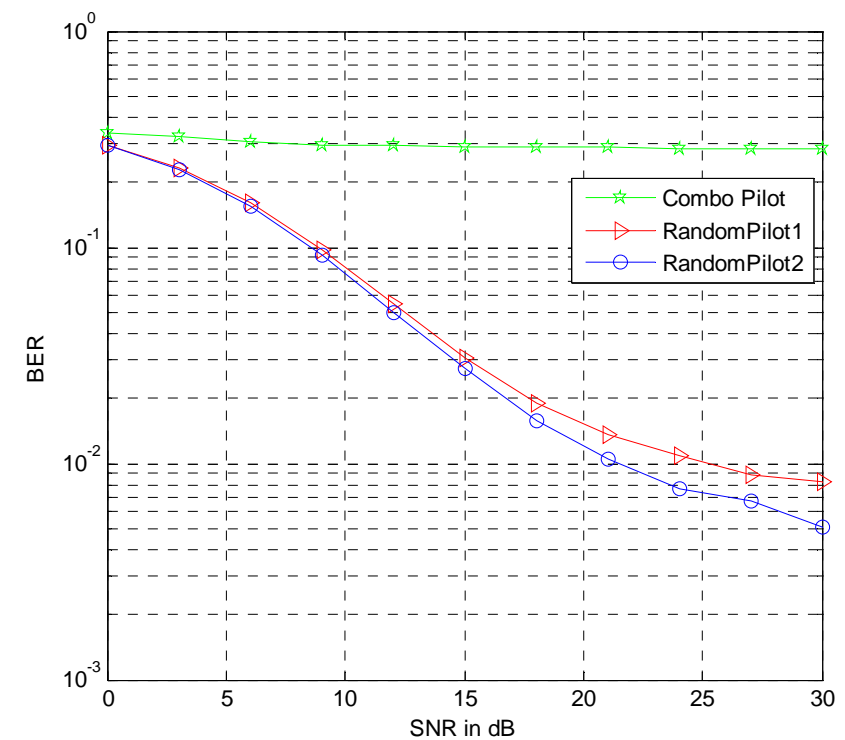

Fig. 5. Bit Error Rate Vs Signal to Noise Ratio using OMP Channel Estimation using Combo and Random Pilot Patterns with 16 pilots 


\section{CONCLUSION AND FUTURE WORK}

Traditional and CS channel estimation techniques have been applied for pilot aided sparse channel estimation in OFDM system. CS algorithm provides better BER performance than traditional algorithms like LS and MMSE algorithms. By exploiting the sparse nature of the wireless channels, CS technique reduces the pilot over head also. The significant improvement in the performance of CS algorithm can be obtained by pilot structure if it achieves the smaller value of mutual coherence. Random pilot generation is not appropriate for practical systems and therefore selection of pilot structure that achieves minimum value of mutual coherence would be promising work for future technologies.

\section{REFERENCES}

[1] Y. Li, "Pilot-Symbol-Aided Channel Estimation for OFDM in Wireless Systems," IEEE Transactions on Vehicular Technology, vol. 49, no.4, pp. 1207 - 1215, Aug. 2000.

[2] M. Hsieh and C. Wei, "Channel estimation for OFDM Systems Based on Comb-Type Pilot Arrangement in Frequency Selective Fading Channels," IEEE Transactions on Consumer Electronics, vol. 44, no.1, pp. 217-225, Feb. 2002.

[3] S. Coleri, M. Ergen, A. Puri and A. Bahai, "Channel Estimation Techniques Based on Pilot Arrangement in OFDM Systems," IEEE Transactions on Broadcasting, vol. 48, no. 3, pp. 223 - 229, Oct. 2002.

[4] Yong Soo Cho, Jeakwonkin, Won Young Yang and Chung G. Kang, MIMO - OFDM Wireless Communication with MATLAB, IEEE Press, John Wiley \& Sons Pvt. Ltd, 2010.

[5] David L. Donoho, “Compressed Sensing," IEEE Transactions on Information Theory, vol.52, no. 4, pp. 1289 - 1306, Apr., 2006.

[6] E.J.Candes, "The Restricted Isometry Property and its Implications for Compressed Sensing," Comptes Rendus Mathematique, vol.346, no.9-10, pp. 589-592, May 2008.

[7] R. Baraniuk, M. Davenport, R. Devore, and M. Wakin, “A Simple Proof of the Restricted Isometry Property for Random Matrices," Constructive Approximation, vol. 28, no. 3, pp. 253-263, Dec.2008.

[8] Georg Taubock and Franz Hlawatsch, "A Compressed Sensing Technique for OFDM Channel Estimation in Mobile Environments: Exploiting Channel Sparsity for Reducing Pilots," Proceedings of IEEE International Conference on Acoustics, Speech and Signal Processing Las Vegas, NV, March-Apr. 2008.

[9] Waheed U. Bajwa, "New Information Processing Theory and Methods for Exploiting Sparsity in Wireless Systems," Ph.D. Dissertation, University of Wisconsin, Madison, WI, 2009.

[10] Chenhao Qi and Lenan Wu, "A Hybrid Compressed Sensing Algorithm for Sparse Channel Estimation in MIMO OFDM Systems," Proceedings of IEEE International Conference on Acoustics, Speech and Signal Processing, Prague,Czech Republic, May 2011, pp. 3488- 3491.

[11] Tian-Ming Ma, Yu-Song Shi, and Ying-Guan Wang, "A Low Complexity MMSE for OFDM Systems over Frequency-Selective Fading Channels,” IEEE Communications Letters, vol. 16, no. 3, pp. 304 -306, Mar. 2012.

[12] Joel A. Tropp and Anna C. Gilbert, "Signal Recovery from Random Measurements Via Orthogonal Matching Pursuit," IEEE Transactions on Information Theory, vol. 53, no. 12, pp.4655-4666, Dec. 2007.

[13] Arvind Ganesh, Zihan Zhou and Yi Ma, "Separation of a Subspace Signal: Algorithms and Conditions," Proceedings of IEEE International Conference on Acoustics Speech Signal Processing, Taipei, Taiwan, Apr. 2009, pp. 3141-3144.

[14] Jian Wang and Byonghyo Shim, "On the Recovery Limit of Sparse Signals using Orthogonal Matching Pursuit", IEEE Transactions on Signal Processing, vol. 60, no.9, pp. 4973 - 4976, Sept. 2012.

\section{Author Profile}

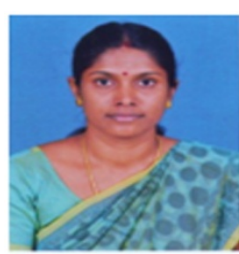

P. Vimala received her Bachelor Degree in Electronics and Communication Engineering from Jayaram College of Engineering, Trichy, Tamil Nadu, India in 2000 and Masters Degree in Process Control and Instrumentation Engineering from Annamalai University, Chidambaram, Tamilnadu, India in 2010. She is currently working as Assistant Professor in the Department of Electronics and Communication Engineering, Faculty of Engineering and Technology, Annamalai University. Her current research areas are Digital Signal Processing, Sparse Signal Processing, Digital Communication and Wireless Communication.

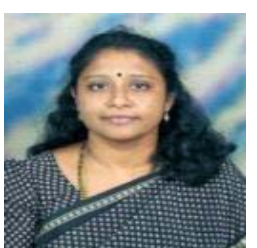

G. Yamuna received her Bachelor Degree in Electronics and Communication Engineering from National Institute of Technology (NIT), Trichy, Tamil Nadu, India in 1987. She received her Master Degree in Power System from Annamalai University in the year 1991. She received her Ph.D. Degree in Electrical Engineering from Annamalai University in 2010. She has published many technical papers in national and international conferences and journals. Currently, she is working as a Professor in the Department of Electronics and Communication Engineering, Faculty of Engineering and Technology, Annamalai University, Tamil Nadu, India. She is an Associate Editor and Reviewer for several National and International Journals. Her research area of interest includes Signal Processing, Image Processing, Wireless Communication Systems, Antenna Design and Information Security. 